\title{
Back to the Future: Therapies for Idiopathic Nephrotic Syndrome
}

\author{
Keisha L. Gibson Dorey Glenn Maria E. Ferris \\ University of North Carolina Kidney Center, Chapel Hill, N.C., USA
}

\section{Key Words}

Nephrotic syndrome · Immunosuppression - Novel

therapies

nologic mechanisms of widely used immunosuppressive therapies may be just as important in palliating proteinuric disease as proposed immunologic functions.

(c) 2015 S. Karger AG, Basel

\section{Abstract}

Background: Roughly $20-40 \%$ of individuals with idiopathic nephrotic syndrome will fail to respond to standard therapies and have a high risk of progression to end stage kidney disease (ESKD). In the last 50 years, no new therapies have been approved specifically for the treatment of these individuals with recalcitrant disease. Summary: Recent in vitro, translational, and clinical studies have identified novel targets and pathways that not only expand our understanding of the complex pathophysiology of proteinuric disease but also provide an opportunity to challenge the tradition of relying on histologic classification of nephrotic diseases to make treatment decisions. Key Messages: The traditional methods of directing the care of individuals with nephrotic syndrome by histological classification or deciding second line therapies on the basis of steroid-responsiveness may soon yield customizing therapies based on our expanding understanding of molecular targets. Important non-immu-

\section{A Look into the Past}

In 1948, a few patients with rheumatoid arthritis were injected with six hormones isolated from adrenal glands by Mayo researcher, Dr. Edward C. Kendall. These patients subsequently developed the resolution of many of their symptoms with one particular hormone. This hormone, later determined to be cortisone, was very cumbersome to isolate. Dr. Hench, a rheumatologist and fellow researcher at Mayo, hypothesized that injecting another substance, adrenocorticotropic hormone, or ACTH, would stimulate the body to produce cortisone and other steroid hormones. Dr. Hench obtained ACTH from the Armour meatpacking company, which was extracting it from pigs as part of an effort to develop markets for leftover animal parts. ACTH was injected into the first rheumatoid arthritis patient in February 1949 with favorable

\section{KARGER 125}

() 2015 S. Karger AG, Basel

0253-5068/15/0393-0105\$39.50/0
Keisha L Gibson, MD, MPH

7005 Burnett Womack

University of North Carolina at Chapel Hill

Chapel Hill, NC 27599-7155 (USA)

E-Mail kgibson@med.unc.edu 
Table 1. Recent trials for the use of ACTH in the treatment of various proteinuric glomerular diseases

\begin{tabular}{lllrc}
\hline Author & Year & Disease & $\mathrm{n}$ & Responders \\
\hline Berg & 2004 & MCD, FSGS, iMN, MSGN & 23 & $43 \%$ \\
Ponticelli & 2006 & iMN & 16 & $88 \%$ \\
Rauen & 2009 & iMN & 4 & $100 \%$ \\
Bomback & 2011 & MCD, FSGS, iMN, IgA & 15 & $40 \%$ \\
Hogan & 2013 & FSGS & 24 & $29 \%$ \\
Hladunewich & 2014 & iMN & 20 & $65 \%$ \\
\hline
\end{tabular}

results. By 1950, thousands of patients, not only those with arthritis but also those with gout, lupus, ulcerative colitis, and many other diseases, had been successfully treated with either cortisone or ACTH $[1,2]$.

The idiopathic nephrotic syndrome affects 5-10 per 100,000 children. Prior to the advent of corticosteroids and antibiotics, the mortality rate for nephrotic syndrome was close to $67 \%$. Following the introduction of sulfonamides in 1944 after World War II, the mortality rate dropped to $\sim 35 \%$ and then even more dramatically in the 1950s after the introduction of ACTH and cortisone [3]. To date, ACTH is the only therapy with FDA approval for the treatment of the nephrotic syndrome.

\section{Old Drugs, New Target}

There has been renewed interest in the role of ACTH for the treatment of primary nephrotic diseases over the last decade. Some of this renewed interest can be attributed to our expanded understanding of melanocortin pathways in glomerular diseases. Podocytes, mesangial cells, glomerular endothelial cells, and renal tubular cells all express melanocortin receptors [4]. Recent studies have reported variable response rates in patients with primary nephrotic syndromes treated with ACTH (table 1) [5-10].

Calcineurin inhibitors such as cyclosporine and tacrolimus have been used to reduce proteinuria in several glomerular diseases. Protein reduction with this therapy has historically been attributed to the downregulation of interleukin 1 and the hemodynamic changes associated with vasoconstriction in which the lower GFR leads to subsequent lowered urinary protein filtration. There is growing data supporting a non-immunologic, non-hemodynamic benefit to calcineurin inhibitors, specifically cyclosporine $[11,12]$. Synaptopodin stabilizes the actin cytoskeleton of the podocyte and is protected from degradation by phosphorylation. Calcineurin dephosphorylates synaptopdin, subjecting it to degradation by cathepsin L and subsequent loss of stabilizing properties [13]. Faul et al. showed that the inhibition of calcineurin by cyclosporine A protected against lipopolysaccharide-induced proteinuria in mice, and also demonstrated that the activation of calcineurin in the podocyte is sufficient to cause proteinuria by causing degradation of synaptopodin [13]. Novel calcineurin substrates such as synaptopodin may provide promising starting points for antiproteinuric drugs that avoid the serious side effects of longterm cyclosporine treatment.

\section{Circulating Factors}

Experimental data to support the existence of soluble mediators that may alter capillary wall permeability in nephrotic syndrome have been reported. A 2001 case report describes the development of high-grade proteinuria and hypoalbuminemia in a 1-day-old, 33-week gestation infant born to a mother who developed proteinuria during the second trimester [14]. The symptoms in this infant resolved after $24 \mathrm{~h}$ without intervention. In a series of 8 patients with FSGS recurrence post-transplant, all developed at least a partial remission with immunoadsorption [15]. These observational findings are supported by translational studies that not only confirmed the ability to induce remission in patients with recurrent FSGS post-transplant with immunoadsorption but also that glomerular permeability could be induced in experimental animals by injecting them with serum from patients with recurrent FSGS [16-18]. Using a galactose affinity purification method to enrich serum from patients with FSGS and FSGS recurrence, Savin et al. showed that cardiotrophin-like cytokine-1 (CLC-1), a member of the IL- 6 cytokine family, is a candidate for the FSGS permeability factor. In this study, they found that CLC-1 was present in the plasma of patients with primary FSGS and in patients with recurrent FSGS in their renal allografts and that its concentration may be up to 100 times that of normal subjects. In addition, they showed that CLC-1 decreases nephrin expression in podocyte culture [16]. Based on the affinity of CLC-1 for galactose, there have been several case reports in the literature on the use of galactose in the treatment of denovo FSGS and recurrence with mixed results. The use of galactose as a novel therapy in the treatment of nephrotic syndrome has been included in an open label 
Table 2. Potential future paradigm for targeted therapy in nephrotic syndrome

\begin{tabular}{lll}
\hline Target & Future therapy & Disease \\
\hline p38 MapK & Losmapimod & FSGS \\
Endothelin receptor & Sparsentan & FSGS \\
B7-1 & Abatacept & FSGS; minimal \\
change disease
\end{tabular}

phase II clinical trial assessing the reduction of proteinuria in patients with FSGS disease resistant to standard therapies [19].

\section{Emerging Therapies}

\section{p38 MAPK Signaling: Losmapimod}

A rapidly developing field of study focuses on the major families of MAPK (or mitogen-activated protein kinases) that translate extracellular stimuli to intracellular responses. The p38 MapK pathway has crucial roles in inflammation, differentiation, senescence, tumorogenesis, and apoptosis. Pro-apoptotic p38 MapK signaling has been implicated in both experimental models of renal injury and in human glomerulopathies. p38 MAPK was found in podocytes and other glomerular cells after disease induction [20]. Inhibition of this signaling pathway was effective in reducing the severity of injury [21]. Increased p38 MAPK activation has been observed in podocytes in biopsies of adults with various forms of nephrotic syndrome [22]. These data provide support for the current multicenter, open-label phase II, proof-of-mechanism study evaluating the effect of losmapimod (a p38 MAPK inhibitor) on the reduction of proteinuria of individuals with corticosteroid or cyclosporine resistant FSGS.

Endothelin Receptor Type 1A antagonism: Sparsentan Signaling crosstalk between glomerular podocytes and endothelial cells has been identified as a factor in progressive glomerulosclerosis. Various mechanisms of podocyte injury activate SMAD proteins associated with EDN1 (Endothelin 1) precursor synthesis and EDN1 release. SMAD proteins are intracellular proteins that transduce extracellular signals from transforming growth factor- $\beta$ ligands to the nucleus to activate downstream gene transcription. Paracrine EDN1/EDNRA (Endothelin receptor) signaling causes mitochondrial oxidative damage and dysfunction in glomerular endothelial cells. Endothelial mitochondrial DNA damage and dysfunction induces podocyte depletion and segmental glomerulosclerosis with nephrotic syndrome, elevated serum creatinine, and eventually decreased survival. The mechanism underlying podocyte apoptosis after endothelial dysfunction has not been elucidated [23]. Researchers have furthermore demonstrated the ability to palliate podocyte effacement and proteinuria in mice with adriamycin-induced podocyte injury treated with sitaxsentan, a selective $\mathrm{ETA}_{1}$ receptor inhibitor [24]. A randomized, doubleblind, controlled study is in progress, comparing the antiproteinuric effects of a dual acting endothelin (A type) and angiotensin II receptors (Type 1) antagonist versus irbesartan (an angiotensin II receptor antagonist) in patients with biopsy demonstrated FSGS [ClinicalTrial.gov NCT01613118].

\section{Biologics: Beyond Rituximab and B cells - \\ Adalimumab and Abatacept}

The role of B-cell depletive therapy with rituximab, a chimeric anti-CD20 monoclonal antibody is yet to be fully determined. Various nonrandomized studies have suggested a favorable response in some patients with idiopathic nephrotic syndrome [25-27]. A recent randomized, double-blinded, placebo-controlled trial of 48 children with frequently relapsing steroid sensitive or steroid-dependent disease showed a significance difference (267 vs. 101 relapse free days [HR 0.27, p < 0.0001]) for patients that received rituximab versus placebo respectively [28]. Studies evaluating its efficacy in patients with steroid and/or calcineurin inhibitor resistant disease have revealed mixed results $[29,30]$. The mechanism of rituximab may not be limited to its role in depleting CD20 positive cells. Recent studies have suggested a novel target for rituximab through podocyte sphingomyelinase-like phosphodiesterase 3b protein (SMPDL-3b), which stabilizes podocyte actin stress fibers and cell survival [31].

Adalimumab is a monoclonal antibody directed against tumor necrosis factor- $\alpha$ (TNF- $\alpha$ ). TNF- $\alpha$ is an inflammatory cytokine produced by mononuclear cells, macrophages, and kidney mesangial cells. Postulated mechanisms of action include recruitment of leukocytes to the site of glomerular injury, induction of cytokines, and growth factors, generation of free oxygen radicals resulting in increased glomerular endothelial cell permeability to albumin, direct cytotoxicity to glomerular mesangial and epithelial cells, and induction of apoptosis. Increases in TNF- $\alpha$ and TNF- $\alpha$ mRNA have been de- 
scribed in FSGS. The role of Adalimumab as a novel therapy for treatment-resistant FSGS was evaluated as an arm in the FONT phase II clinical trial [19]. The results from this trial have not yet been published.

Abatacept is a monoclonal antibody that blocks CD80 T-cell signaling. Protein B7-1 (CD80) is commonly found on antigen presenting cells. This ligand functions as a co-stimulatory signal for T-cells depending on which ligand it binds to. Binding with CD28 creates a stimulatory signal while binding with CTLA-4 promotes a regulatory signal. B7-1 is not expressed by normal podocytes but has been found in human podocytes with various glomerulonephritidies including FSGS and MPGN. Increased podocyte expression of B7-1 has additionally been found in mouse models of proteinuria [32]. Yu et al. reported that B7-1 influences podocyte structure and function. They demonstrated that increased podocyte migration and proteinuria was associated with intact B7-1 expression in their in vitro studies. Their work further found that B7-1 binds to $\beta 1$ integrin, which is an essential ligand that binds podocytes to the glomerular basement membrane and interacts with the actin cytoskeleton [33]. Abatacept is a fusion protein of the Fc re- gion of the immunoglobulin IgG1 and extracellular domain of CTLA-4. It blocks CD80 signaling through competitive bidding for $\mathrm{T}$-cell ligands and has been proven safe and effective for patients with rheumatoid arthritis. $\mathrm{Yu}$ et al. were not only able to demonstrate in their in vitro studies that Abatacept prevented B7-1 induced podocyte migration and prevented B7-1/31 integrin association, but also that they could reduce proteinuric remission in 5 patients with FSGS that had failed therapy with rituximab.

\section{Conclusions}

There is renewed hope for the $25-40 \%$ of individuals with primary nephrotic diseases that fail to respond to conventional therapies. Whether considering new targets for old therapies or new drugs designed against new targets, the armamentarium for therapies we offer our patients with idiopathic nephrotic syndrome is expanding. This growing body of therapies will enhance our ability to improve the outcomes and reduce the morbidity for a patient population that has historically been difficult to manage.

\section{References}

$>1$ Sprague RG: Summary: cortisone and ACTH in clinical medicine. Proc Staff Meet Mayo Clin 1950;25:500-502.

$>2$ Sprague RG, Power MH, et al: Observations on the physiologic effects of cortisone and ACTH in man. Arch Intern Med (Chic) 1950; 85:199-258.

-3 Barnett HL, et al: The effects of ACTH and cortisone on the nephrotic syndrome. AMA Am J Dis Child 1950;80:519-520.

-4 Gong R: Leveraging melanocortin pathways to treat glomerular diseases. Adv Chronic Kidney Dis 2014;21:134-151.

$>5$ Berg AL, Arnadottir M: ACTH-induced improvement in the nephrotic syndrome in patients with a variety of diagnoses. Nephrol Dial Transplant 2004;19:1305-1307.

6 Hogan J, et al: Treatment of idiopathic FSGS with adrenocorticotropic hormone gel. Clin J Am Soc Nephrol 2013;8:2072-2081.

$>7$ Bomback AS, Radhakrishnan J: Treatment of nephrotic syndrome with adrenocorticotropic hormone (ACTH). Discov Med 2011;12: 91-96.

$>8$ Ponticelli C, et al: A randomized pilot trial comparing methylprednisolone plus a cytotoxic agent versus synthetic adrenocorticotropic hormone in idiopathic membranous nephropathy. Am J Kidney Dis 2006;47:233240.
$>9$ Rauen T, et al: Case series of idiopathic membranous nephropathy with long-term beneficial effects of ACTH peptide 1-24. Clin Nephrol 2009;71:637-642.

10 Hladunewich MA, et al: A pilot study to determine the dose and effectiveness of adrenocorticotrophic hormone (H.P. Acthar ${ }^{\circledR} \mathrm{Gel}$ ) in nephrotic syndrome due to idiopathic membranous nephropathy. Nephrol Dial Transplant 2014;29:1570-1577.

11 Zietse R, et al: Effects of cyclosporin A on glomerular barrier function in the nephrotic syndrome. Clin Sci (Lond) 1992;82:641650 .

12 Meyrier A: Antiproteinuric and immunological effects of cyclosporin A in the treatment of glomerular diseases. Nephrol Dial Transplant 1992;(7 suppl 1):80-84.

13 Faul C, et al: The actin cytoskeleton of kidney podocytes is a direct target of the antiproteinuric effect of cyclosporine A. Nat Med 2008; 14:931-938.

14 Kemper MJ, Wolf G, Muller-Wiefel DE: Transmission of glomerular permeability factor from a mother to her child. N Engl J Med 2001;344:386-387.

15 Dantal J, et al: Effect of plasma protein adsorption on protein excretion in kidneytransplant recipients with recurrent nephrotic syndrome. N Engl J Med 1994;330:7-14.
16 Savin VJ, et al: Galactose binds to focal segmental glomerulosclerosis permeability factor and inhibits its activity. Transl Res 2008; 151:288-292.

17 Savin VJ, et al: Circulating factor associated with increased glomerular permeability to albumin in recurrent focal segmental glomerulosclerosis. N Engl J Med 1996;334:878-883.

18 Sharma M, et al: Proteinuria after injection of human focal segmental glomerulosclerosis factor. Transplantation 2002;73:366-372.

19 Trachtman H, et al: Novel therapies for resistant focal segmental glomerulosclerosis (FONT) phase II clinical trial: study design. BMC Nephrol 2011;12:8.

20 Stambe C, et al: Blockade of p38alpha MAPK ameliorates acute inflammatory renal injury in rat anti-GBM glomerulonephritis. J Am Soc Nephrol 2003;14:338-351.

21 Sheryanna A, et al: Inhibition of p38 mitogenactivated protein kinase is effective in the treatment of experimental crescentic glomerulonephritis and suppresses monocyte chemoattractant protein-1 but not IL-1beta or IL-6. J Am Soc Nephrol 2007;18:1167-1179.

22 Koshikawa M, et al: Role of p38 mitogen-activated protein kinase activation in podocyte injury and proteinuria in experimental nephrotic syndrome. J Am Soc Nephrol 2005;16: 2690-2701. 
23 Daehn I, et al: Endothelial mitochondrial oxidative stress determines podocyte depletion in segmental glomerulosclerosis. J Clin Invest 2014;124:1608-1621.

24 Buelli S, et al: Beta-arrestin-1 drives endothelin-1-mediated podocyte activation and sustains renal injury. J Am Soc Nephrol 2014;25: 523-533.

25 Ravani P, et al: Rituximab is a safe and effective long-term treatment for children with steroid and calcineurin inhibitor-dependent idiopathic nephrotic syndrome. Kidney Int 2013;84:1025-1033.

26 Ruggenenti P, et al: Rituximab in steroid-dependent or frequently relapsing idiopathic nephrotic syndrome. J Am Soc Nephrol 2014; 25:850-863.
27 Bruchfeld A, et al: Rituximab for minimal change disease in adults: long-term followup. Nephrol Dial Transplant 2014;29:851856.

28 Iijima K, et al: Rituximab for childhood-onset, complicated, frequently relapsing nephrotic syndrome or steroid-dependent nephrotic syndrome: a multicentre, doubleblind, randomised, placebo-controlled trial. Lancet 2014;384:1273-1281.

29 Kamei K, et al: Rituximab treatment combined with methylprednisolone pulse therapy and immunosuppressants for childhood steroid-resistant nephrotic syndrome. Pediatr Nephrol 2014;29:1181-1187.
30 Sinha A, et al: Efficacy and safety of rituximab in children with difficult-to-treat nephrotic syndrome. Nephrol Dial Transplant 2014;pii: gfu267, Epub ahead of print.

31 Perosa F, et al: Generation of biologically active linear and cyclic peptides has revealed a unique fine specificity of rituximab and its possible cross-reactivity with acid sphingomyelinase-like phosphodiesterase $3 \mathrm{~b}$ precursor. Blood 2006;107:1070-1077.

32 Reiser J, et al: Induction of B7-1 in podocytes is associated with nephrotic syndrome. J Clin Invest 2004;113:1390-1397.

$33 \mathrm{Yu}$ CC, et al: Abatacept in B7-1-positive proteinuric kidney disease. N Engl J Med 2013; 369:2416-2423. 